Franciele Camila LUCHESE ${ }^{1}$ Marivanda PERIN ${ }^{1}$

Rafael Sbicigo AITA ${ }^{1}$

Vanessa Daniele MOTTIN ${ }^{1}$

Marcelo Beltrão MOLENTO²

Silvia Gonzalez MONTEIRO ${ }^{2}$

Correspondênciapara:

MARCELOBELTRÃOMOLENTO

Laboratório de Doenças Parasitárias

Departamento de Medicina Veterinária

Universidade Federal do Paraná

SCAVUFPR

Rua dos Funcionários, 1540-Juvevê

80035-050 - Curitiba-PR

molento@ufpr.br

Recebido para publicação: 25/01/2005 Aprovado para publicação: 07/02/2007

\title{
Prevalência de espécies de Eimeria em frangos de criação industrial e alternativa
}

\author{
1 - Curso de Medicina Veterinária da Universidade Federal de Santa Maria \\ Santa Maria - RS \\ 2 - Setor de Doenças Parasitárias da Universidade Federal de Santa Maria, \\ Santa Maria - RS
}

\section{Resumo}

As eimerioses causam grave problema às criações de frangos, como, redução do ganho de peso e aumento da conversão alimentar, gerando grandes perdas econômicas. O objetivo deste trabalho foi determinar a prevalência e espécies de Eimeria sp. em dois grupos, um de criação industrial com a linhagem Hubbard tratado com Premix contendo medicamentos coccidiostáticos até sete dias antes do abate e um de frango de criação alternativa com a linhagem Redro Plumé sem tratamento. Após análise parasitológica de 100 amostras $(n=50 /$ grupo) comprovou-se a infecção mista de sete espécies: Eimeria tenella, E. necatrix, E. acervulina, E. maxima, E. brunetti, E. mitis, E. mivati, em ambos os grupos. Ocorreu distribuição normal de oocistos somente no grupo comercial. O número de oocistos encontrado na segunda amostra do grupo tratado foi estatisticamente diferente da primeira $(\mathrm{P}=0,023)$ e da primeira $(\mathrm{P}=0,016)$ e da segunda colheita $(\mathrm{P}=0,028)$ do grupo sem tratamento. E. maxima foi a espécie mais prevalente no grupo tratado e sem tratamento.

\section{Introdução}

O desenvolvimento tecnológico empregado na avicultura industrial melhorou sobremaneira os índices de conversão alimentar e precocidade das aves ao abate. O emprego desta tecnologia está associado ao aumento no consumo da carne de frango no Brasil e no mundo nesta última década. Os protozoários do gênero Eimeria, também conhecidos como coccídeos, são a principal parasitose na avicultura, tanto industrial, como rural ou peri-urbana, causando graves prejuízos econômicos, principalmente devido a episódios de diarréia e mortes em animais jovens. Castro ${ }^{1}$ considera que estes prejuízos possam chegar a US\$19 milhões/ anuais no Brasil. Williams ${ }^{2}$ determinou que o custo anual causado por coccídeos é de aproximadamente US\$21,5 milhões na Inglaterra. Sendo 80\% ocasionado pela perda de peso e mortalidade e $17 \%$ devido ao custo da terapia. No mundo, as perdas podem chegar a aproximadamente US $\$ 1,5$ bilhão/ano ${ }^{3}$.

As eimerioses apresentam caráter endêmico nas granjas. Sete espécies podem ser encontradas de forma prevalente em aves comerciais ou não: Eimeria acervulina, E. tenella, E. necatrix, E. brunitti, E. maxima, E. mitis, E. mivati. Al-Natour, Suleiman e Abo-Shehada ${ }^{4}$ determinaram que $50 \%$ das propriedades $(\mathrm{n}=200)$ na Jordânia possuem as sete espécies. Sendo E. tenella, E. necatrix, E. brunitti e E. maxima encontradas em 39, 12, 12 e $10 \%$ das granjas. Reza Razmi e Kalideri ${ }^{5}$ encontraram E. acervulina, E. maxima e E. tenella em 97, 41 e 12\% no Irã.

Muito embora existam outras estratégias de controle destas infecções, a utilização de compostos químicos é o método mais comum e eficiente no controle da coccidiose em granjas comerciais. Estes medicamentos são administrados desde o primeiro dia de vida das aves até sete dias antes do abate, respeitando o período de 
carência dos compostos no organismo animal. Atualmente existe uma tendência mundial pela produção de alimentos, visando o máximo conforto dos animais e a não utilização de produtos químicos. Esta nova parcela de mercado tem despertado grande interesse em consumidores e produtores aos possíveis riscos à saúde humana decorrente da utilização indiscriminada de produtos antibióticos e quimioterápicos nas aves comerciais ${ }^{6}$. Uma opção que está sendo difundida internacionalmente é a produção de frangos orgânicos e/ou alternativos onde é vetada a utilização de drogas com ação coccidiostáticas. A utilização destes compostos só é permitida após comprovação clínica.

Devido à importância da coccidiose aviária, o objetivo deste trabalho foi determinar a prevalência da infecção por estes organismos em nível de espécie em dois grupos de frango de corte oriundos de criação industrial, tratados com coccidiostáticos diariamente e frangos de criação alternativa sem tratamento para coccidiose no momento pré-abate.

\section{Material e Métodos}

\section{Descrição da criação comercial - industrial}

Os pintos chegam a granja com $45 \mathrm{~g}$ e são alimentados com ração específica até atingirem o peso ideal aos 38-42 dias, com um ganho médio diário (GMD) de 45g. A cama onde os animais permanecem é composta por raspa de madeira e é trocada uma vez ao ano, sendo que a cada lote novo ocorre uma reposição parcial desta. As aves são abrigadas em uma densidade de 10 aves $/ \mathrm{m}^{2}$. Os bebedouros utilizados são do tipo nipple com 15 aves/bebedouro. As aves recebem Premix medicado que é fornecido pela companhia integradora, oferecida ad libitum em comedouros automáticos (55 aves/comedouro). A droga utilizada e sua concentração foram mantidas sob sigilo industrial. Os técnicos responsáveis relataram mortalidade pré-abate de $2 \%$ no grupo estudado.

Descrição da criação alternativa

As técnicas de criação do frango alternativo não utilizam promotores artificiais de crescimento e são livres de antibióticos. A ração para frangos é elaborada com grãos tipo 1 de milho e/ou soja de alta qualidade e complementada com vitaminas e minerais, sem a inclusão de ingredientes de origem animal. Estes ingredientes podem ser adquiridos sem restrições de produção como é o caso da criação orgânica. A água é fornecida ad libitum. As aves levam mais tempo para atingir peso semelhante do que em criações industrias, atingindo $2,5 \mathrm{~kg}$ aos 80 dias com GMD de $27 \mathrm{~g}$. As aves são colocadas em uma concentração de $5-6 \mathrm{~m}^{2}$ de pastagem/ave e nos galpões a lotação é de 12 aves $/ \mathrm{m}^{2}$. A cama é semelhante à criação comercial, no entanto esta é trocada totalmente antes da entrada de um novo lote. Os episódios de eimeriose são tratados através da adição de $250 \mathrm{~g}$ de sulfa em 1000 litros de água, juntamente com penicilina e enrofloxacina na água por sete dias. Foi relatada mortalidade de $2,5 \%$ no grupo estudado.

Colheita das amostras e identificação das espécies Foram colhidas 50 amostras fecais de frangos industriais e 50 amostras de frangos de criação alternativa, em duas ocasiões, provenientes dos municípios de Lajeado e Agudo, respectivamente, no estado do Rio Grande do Sul. Ambas as instalações fazem parte de um programa de granjas integradas e comportam 10.000 aves por período. As amostras foram tomadas totalmente ao acaso no momento pré-abate. As amostras fecais foram acondicionadas em recipiente térmico até a realização das técnicas parasitológicas. A contagem dos oocistos foi realizada através da técnica de Gordon e Whitlock ${ }^{7}$ modificada. Vinte amostras ( $n=10 /$ grupo) foram acondicionadas em Placa de Petri contendo solução de Bicromato de Potássio a 2,5\% e mantidas em temperatura ambiente para esporulação e identificação das espécies. Dois mil oocistos esporulados foram identificados após mensuração das medidas do diâmetro polar (DP) e diâmetro equatorial (DE) para 
Tabela 1 - Média e desvio padrão (log) da contagem de oocistos (OPG) de Eimeria sp. proveniente de duas colheitas em frangos de corte de criação comercial e de criação alternativa

\begin{tabular}{lcc}
\hline & \multicolumn{2}{c}{ OPG } \\
\hline Tipo de criação & Amostra 1 & Amostra 2 \\
Comercial & $3,700(0,205)$ a C & $3,783(0,147)$ b D \\
Alternativa & $3,590(0,535)$ a C & $3,609(0,531)$ a E \\
\hline
\end{tabular}

Letras minúsculas diferentes na mesma linha significam diferença estatística $(P<0,05)$. Letras maiúsculas na mesma coluna significam diferença estatística $(\mathrm{P}<0,05)$

posterior cálculo do índice morfométrico $(\mathrm{IM}=\mathrm{DP} / \mathrm{DE})$.

\section{Análise estatística}

O delineamento experimental utilizado foi inteiramente casualizado. Os dados foram transformados em escala logarítmica (log-10) e submetidos ao desvio padrão e à análise pelo teste " $\vec{\imath}$ " de Student ao nível de 5\% de significância. As análises foram realizadas utilizando-se o programa Microsoft EXCEL.

\section{Resultados}

Foi determinado que o sistema de criação alternativa apresentou uma maior média absoluta de oocistos $(x=7.587)$ quando comparado com o grupo industrial $(x=5.996)$. Apesar do uso de drogas coccidiostáticas na ração do grupo industrial, foi observada alta prevalência de coccídeos nas duas amostragens (Tabela 1). Ocorreu semelhança na prevalência de espécies entre grupos e entre períodos amostrais. Foram identificadas sete espécies de Eimeria spp.: E. maxima, E. brunetti, E. tenella, E. acervulina, E. mitis, E. mivati e E. necatrix. As espécies identificadas com maior freqüência no grupo de frangos industriais na primeira colheita foram: E. maxima (44\%), E. brunetti (18\%) e E. tenella $(13,34 \%)$ e na segunda colheita foi: E. maxima (45,52\%), E. acervulina $(12,32 \%)$ e E. tenella $(14 \%)$. No grupo de criação alternativa foi identificado: E. maxima $(53,60$ e $42 \%)$, E. brunetti $(13,40$ e $20 \%$ ) e E. tenella $(15,80$ e $14 \%)$ na primeira e na segunda colheita respectivamente. As demais espécies obtiveram percentual inferior a $8 \%$.
Foi observada diferença significativa $(\mathrm{P}=0,023)$ entre a média de oocistos de Eimeria spp. da primeira e segunda colheita do grupo industrial. A ocorrência de oocistos pode ser devido às falhas no manejo sanitário e a alta contaminação ambiental (dados não publicados). A média de oocistos entre a primeira e a segunda colheita do grupo de criação alternativa não apresentou diferença significativa $(\mathrm{P}=0,864)$. A comparação entre a primeira colheita do grupo industrial, com a primeira colheita do grupo alternativo revelou que não houve diferença significativa $(\mathrm{P}=0,180)$. A segunda colheita do grupo industrial foi significativamente diferente da primeira $(\mathrm{P}=0,016)$ e da segunda colheita do grupo alternativo $(\mathrm{P}=0,028)$ (Tabela 1$)$. Observou-se s distribuição normal somente no grupo industrial, em ambas as colheitas de material (Gráfico 1).

Pode-se supor que existam falhas na higienização dos galpões entre os lotes ${ }^{8}$, na informação das pessoas envolvidas com a atividade e na eficácia dos produtos utilizados. O surgimento de populações resistentes a estes produtos é devido ao uso intensivo das drogas, a sua rápida alternância e ao aumento da concentração dos produtos nas rações medicadas. Muito embora Permin e Hansen ${ }^{9}$ sugiram que se poderia evitar o desenvolvimento da resistência parasitária através da alternância rápida de produtos químicos, esta estratégia deve ser evitada. Independentemente do mecanismo de ação ou espectro, esta forma de controle pode promover a resistência lateral e cruzada como já foi determinado em ruminantes ${ }^{10}$. Entretanto, Daugschies, Gasslein e Rommel ${ }^{11}$ observaram também que, na maioria das 
vezes, pode ocorrer pouca disponibilidade do princípio ativo no animal, manifestando uma redução da eficácia do tratamento, sem que ocorra resistência.

Em relação ao caráter farmacológico dos produtos, espera-se que qualquer aditivo administrado as aves seja eliminado ou perca o efeito em um período menor possível. McDougald e Seibert ${ }^{12}$ determinaram o período de persistência de sete drogas coccidiostáticas com poder residual variável (diclazuril, monensin, salinomicina, halofuginone, nicarbazin, robenidine, amprolium e lasalocid) comumente utilizadas nos Estados Unidos. Cada produto foi testado contra uma infecção mista de Eimeria spp. em grupos de 10 animais. Os animais apresentaram vários graus de lesões intestinais com perda de peso e somente o diclazuril manteve ação residual efetiva após 7 dias. No presente trabalho, foi determinado que a medicação fornecida às aves apresentou curto período de persistência, inferior a 3 dias. $O$ fornecimento da medicação diária pode ter prejudicado o desenvolvimento da imunidade nestes animais, visto que se espera um aumento da resposta imune após o contato com o organismo agressor que ocorre nas primeiras semanas de vida do animal ${ }^{13}$. Desta forma, a proteção química no grupo industrial, pode ter causado uma supressão da resposta imune, manifestada através da curva normal. Supõe-se que o grupo de criação alternativa tenha manifestado alto grau de infecção no início do ciclo de produção com conseqüente redução na produção de oocistos após este.

Embora a manutenção da sanidade avícola possa ser comprometida com a restrição do uso de medicação, as crescentes manifestações quanto aos riscos da utilização de anticoccidianos na cadeia alimentar têm desencadeado pesquisas com o intuito de desenvolver novas alternativas. Vários fitoterápicos foram utilizados para determinar a eficácia no controle de E. tenella, em aves de um dia, infectadas com $1 \times 10^{5}$ oocistos. A solução foi colocada na água de bebida das aves e após cinco dias, foi medido, grau de sobrevivência, severidade das lesões, ganho de peso, presença de sangue nas fezes e contagem de oocistos. Sophora flavescens, Pulsatilla koreana, Sinomenium acutum, Ulmus macrocarpa e Quisqualis indica apresentaram resultados satisfatórios, sendo observada total ausência de sinais de infecção no grupo Sophora flavescens ${ }^{14}$. Biavatti et al. ${ }^{15}$ determinaram que Alternanthera brasiliana (180 ml/200 kg de ração) e extrato de própolis ( $200 \mathrm{ml} / 200 \mathrm{~kg}$ de ração) foram capazes de obter ganho de peso compensatório, comparado ao grupo

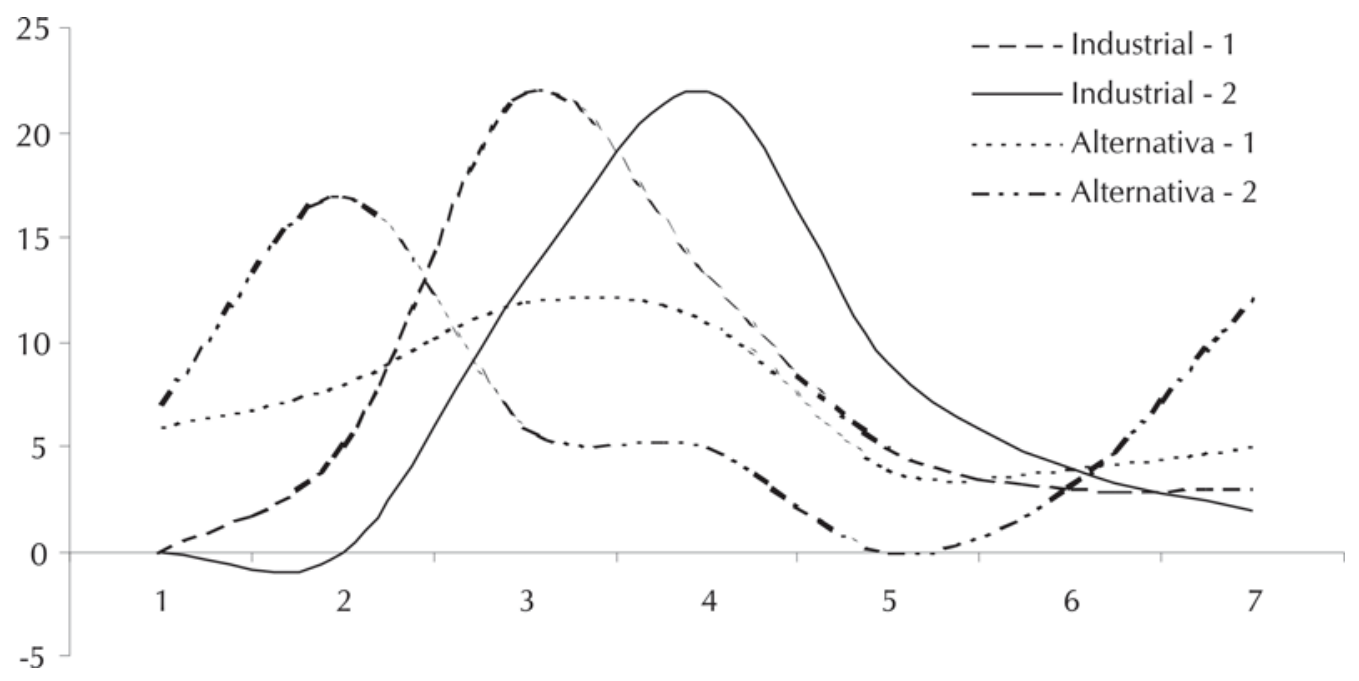

Gráfico 1 - Distribuição de oocistos (log-10) de Eimeria sp. em frangos de criação industrial ealternativa provenientes de duas colheitas 
tratado com drogas coccidiostáticas, em 600 pintos, inoculados com E. acervulina/ave. Acredita-se que este fato seja devido às propriedades antimicrobianas destes produtos, resultando na recuperação das lesões intestinais.

\section{Conclusões}

Conclui-se que frangos de criação industrial e alternativa podem ser portadores de infecção com até sete espécies Eimeria sppp. no Brasil. $\mathrm{O}$ estudo também revelou que o uso de drogas coccidiostáticas na ração de frangos na forma de Premix, não elimina a possibilidade de existir infecções mistas por coccídeos, possivelmente, devido ao curto poder residual dos princípios utilizados e a alta taxa de prevalência de oocistos. As estratégias sanitárias adotadas na criação industrial podem apresentar falhas no controle da eimeriose, possibilitando a contaminação entre lotes. A ocorrência de Eimeria sp. na criação alternativa não inviabiliza a atividade. Embora com menor prevalência em aves tratadas do que em aves que não receberam tratamento, E. maxima foi a espécie mais prevalente. Acreditasse que o contato dos animais com oocistos no meio ambiente estimule a resposta imune de forma compensatória. O caráter isolado das diferentes linhagens deve ser avaliado sob as mesmas condições de criação para determinar qual a influência da capacidade genética frente à infecção mista de Eimeria spp.

\section{Agradecimentos}

Os autores agradecem os responsáveis técnicos das granjas envolvidas neste estudo pelo fornecimento de informações necessárias para a conclusão deste trabalho.

\section{Prevalence of Eimeria species in industrial and alternative bred chicken}

\section{Abstract}

Eimeria infection causes a great problem in chicken, such as, reduction in weight gain and increase in feed conversion, with significant economic losses. The objective of this study was to determine the prevalence and the species of Eimeria sp. in two groups, one group from industrial raised Hubbard line treated with Premix with coccidiostatic drugs 7 days before slaughter and one group from alternative raised Redro Plumé line without treatment. Parasitologic analysis were done on 100 samples ( $\mathrm{n}=50$ /group) with the diagnose of a mix infection of seven species: Eimeria tenella, E. necatrix, E. acervulina, E. maxima, E. brunetti, E. mitis, E. mivati, in both groups. There was a normal distribution of oocists only in the industrial group. Oocists found in the second sampling was statistically different from the first sampling $(\mathrm{P}=0,023)$ and from the first $(\mathrm{P}=0,016)$ and from the second sampling $(\mathrm{P}=0,028)$ of the alternative group. $E$. maxima was the most prevalent species in the treated and untreated groups.

\section{Referências}

1 CASTRO, A. G. M. Situação atual da coccidiose no Brasil. Importância econômica. In: SIMPÓSIO INTERNACIONAL DE COCCIDIOSE, 1., Santos, 1994. Anais.. Santos: Fundação APINCO de Ciência e Tecnologia Avícola, 1994. p. 45-54.

2 WILLIAMS, R. B. A compartmetalised model for the estimation of the cost of coccidiosis to the world's chicken production industry. International Journal for Parasitology, v. 29, n. 8, p. 1209-1229, 1999.

3 MCDOUGALD, L.; REID, W. Coccidiosis. In: CALNEK, B.; BARNES, H.; BEARD, C.; MCDOUGALD, L.; SAIF, Y. Disease of poultry. $10^{\circ}$ ed. lowa: Iowa State University Press, 1997. p. 865-83.

4 AL-NATOUR, M. Q.; SULEIMAN, M. M.; ABO- 
SHEHADA, M. N. Flock-level prevalence of Eimeria species among broiler chicks in northern Jordan. Preventive Veterinary Medicine, v. 53, n. 4, p. 305310, 2002.

5 REZA RAZMI, G.; KALIDERI, G. A. Prevalence of subclinical coccidiosis in broiler-chicken farms in the municipality of Mashhad, Khorasan, Iran. Preventive Veterinary Medicine, v. 44, n. 3-4, p. 247-253, 2000.

6 FILHO, L. C. D.; MENDES, C. M. I. Viabilidade Técnica e Econômica na Criação alternativa de frangos. In: CONGRESSO APINCO DE CIÊNCIA E TECNOLOGIA AVÍCOLA. 20., Campinas, 2001. Anais... Campinas: Fundação APINCO de Ciência e Tecnologia Avícola, 2001. p. 255-266.

7 GORDON, H. M.; WHITLOCK, H. V. A new technique for courting nematode eggs in sheep faeces. Journal of The Council of Science, Industry and Research in Australia, v. 12, p. 50-52, 1939

8 COSTA, C. A. F.; ÁVILA, V. S. Efeito da idade das aves e da reutilização e manejo da cama do aviário sobre a coccidiose em frangos de corte. Arquivo Brasileiro de Medicina Veterinária e Zootecnia, v. 48, n. 4, p. 403413, 1996.

9 PERMIN, A.; HANSEN, J. W. Epidemiology, diagnosis and control of poultry parasites. Roma: Food and Agriculture Organization / United Nations, 1998. 160

10 MOLENTO, M. B. Multidrug resistance in Haemonchus contortus associated with suppressive treatment and rapid drug alternation. Revista Brasileira de Parasitologia Veterinária, v. 13, p. 272, 2004.

11 DAUGSCHIES, A.; GASSLEIN, U.; ROMMEL, M. Comparative efficacy of anticoccidials under the conditions of commercial broiler production and in battery trials. Veterinary Parasitology, v. 76, n. 3, p. 163-171, 1998.

12 MCDOUGALD, L.; SEIBERT, B. P. Residual activity of anticoccidial drugs in chickens after withdrawal of medicated feeds. Veterinary Parasitology, v. 74, n. 2/4, p. 91-99. 1998.

13 RUFF, M. D. Important parasites in poultry production systems. Veterinary Parasitology, v. 84, n. 3-4, p. 337-347, 1999

14 YOUN, H. J.; NOH, J. W. Screening of the anticoccidial effect of the herb extracts against Eimeria tenella. Veterinary Parasitology, v. 96, n. 4, p. 257263, 2001.

15 BIAVATTI, M. W. et al. Preliminary studies of alternative feed additives for broilers: Alternanthera brasiliana extract, propolisextract and linseed oil. Revista Brasileira de Ciência Avícola, v. 5, p. 147-151, 2003. 